\title{
A Laminar Flow Model for Mucous Gel Transport in a Cough Machine Simulating Trachea: Effect of Surfactant as a Sol Phase Layer
}

\author{
Dipak Kumar Satpathi, Addepalli Ramu \\ Department of Mathematics, BITS Pilani Hyderabad Campus, Hyderabad, India \\ Email: dipak@hyderabad.bits-pilani.ac.in, aramu@hyderabad.bits-pilani.ac.in
}

Received June 3, 2013; revised July 3, 2013; accepted July 10, 2013

Copyright (C) 2013 Dipak Kumar Satpathi, Addepalli Ramu. This is an open access article distributed under the Creative Commons Attribution License, which permits unrestricted use, distribution, and reproduction in any medium, provided the original work is properly cited.

\begin{abstract}
In this paper, a planar three layer quasi-steady laminar flow model is proposed in a cough machine which simulates mucous gel transport in model trachea due to mild forced expiration. The flow is governed by the time dependent pressure gradient generated in trachea due to mild forced expiration. Mucous gel is represented by a viscoelastic Voigt element whereas sol phase fluid and air are considered as Newtonian fluids. For fixed airflow rate, it is shown that when the viscosity of mucous gel is small, mucous gel transport decreases as the elastic modulus increases. However, elastic modulus has negligible effect on large gel viscosity. It is also shown that for fixed airflow rate and fixed airway dimension, mucous gel transport increases with the thickness of sol phase fluid and this increase is further enhanced as the viscosity of sol phase fluid decreases. The effect of surfactant is studied by considering sol phase as surfactant layer which causes slip at the wall and interface of sol phase and mucous gel. It is found that in the presence of surfactant mucous gel transport is enhanced.
\end{abstract}

Keywords: Mucous Gel; Sol Phase; Surfactant; Laminar Flow; Trachea; Voigt Element

\section{Introduction}

Mucociliary clearance is an important pulmonary defense mechanism that serves to remove inhaled substances from the lung. It depends upon the relationship between cilia, mucus and periciliary fluid. The mucociliary function is depressed by a variety of water soluble atmospheric pollutants such as $\mathrm{SO}_{2}$ and $\mathrm{NO}_{2}$ [1]. The presence of surfactant in the mucoserous lining of airways helps in increasing the mucus transport and has been investigated experimentally [2-5]. It was pointed out that surfactant caused relative increase in transport rate [2]. It was also showed that in presence of surfactant mucus transport is more $[3,5]$. Bronchial surfactant is essential for bronchoalveolar transport mechanisms including ciliary and non-ciliary mucus transport [4]. In [5], Rubin et al. showed that surfactant therapy appears to improve mucus clearability.

In the case of pulmonary diseases (cystic fibrosis, chronic bronchitis, etc.) excessive amount of mucus is formed in the respiratory tract, which is transported mainly by coughing or forced expiration. This transport also depends upon the depths of mucus and serous layers and the rheological properties of mucus [6]. Mucus transport in a cough machine has been studied by a group of investigators under external applied pressure gradient [6-13]. In [7,8], Scherer and Burtz conducted fluid mechanical experiments relevant to coughing, using air and liquid blown out of a straight tube by turbulent jet. They showed that the liquid transport decreases as the viscosity of liquid increases by assuming that the flow is quasi-steady and turbulent stress of air is equal to viscous stress in the liquid. In [9-11], King and co-investigators in their experiments have shown that the transport increases with the increase in the thickness of mucous gel air flow rate and with the decrease in its elastic modulus. It was observed that mucous gel transport in a simulated cough machine increases as the viscosity of serous layer simulates decreases $[6,12,13]$.

It may be noted that no mathematical model is developed so far to explain the above experimental observations, particularly with surfactant as a sol phase layer. In view of this, in this paper, we present a quasi-steady state three layer laminar flow model (mucous gel as viscoelas- 
tic Voigt element, air and surfactant sol phase fluid as Newtonian fluids) for mucous gel transport in a cough machine simulating trachea by considering the surfactant sol phase as serous layer. Due to the presence of surfactant, the slip effects at the boundaries of the surfactant layer are taken into account in the model. It is assumed that the gel transport is caused by a time dependent pressure gradient due to mild forced expiration.

\section{Modelling and Solution}

We consider the quasi-steady state simultaneous laminar flow of surfactant sol phase fluid, viscoelastic mucous gel and air in a rectangular channel, relevant to mucous gel transport in a cough machine simulating a model trachea. The flow assumed to be caused by a time dependent pressure gradient generated by air motion simulating mild forced expiration in trachea. The flow geometry is shown in Figure 1, where surfactant sol phase fluid $\left(0 \leq y \leq h_{s}\right)$, mucous gel $\left(h_{s} \leq y \leq h_{m}\right)$ and air $\left(h_{m} \leq y \leq h_{a}\right)$ regions are indicated.

The equations governing the laminar flow of surfactant sol phase fluid, viscoelastic mucous gel and air under quasi-steady state condition can be written as follows:

Region I $\left(0 \leq y \leq h_{s}\right)$ surfactant sol phase

$$
-\frac{\partial p}{\partial x}+\frac{\partial \tau_{s}}{\partial y}=0, \tau_{s}=\mu_{s} \frac{\partial u_{s}}{\partial y}
$$

Region II $\left(h_{s} \leq y \leq h_{m}\right)$ mucous gel

$$
\begin{gathered}
-\frac{\partial p}{\partial x}+\frac{\partial \tau_{m}}{\partial y}=0 \\
\frac{\partial \tau_{m}}{\partial t}=G \frac{\partial u_{m}}{\partial y}+\mu_{m} \frac{\partial}{\partial t}\left(\frac{\partial u_{m}}{\partial y}\right)
\end{gathered}
$$

Region III $\left(h_{m} \leq y \leq h_{a}\right)$ air

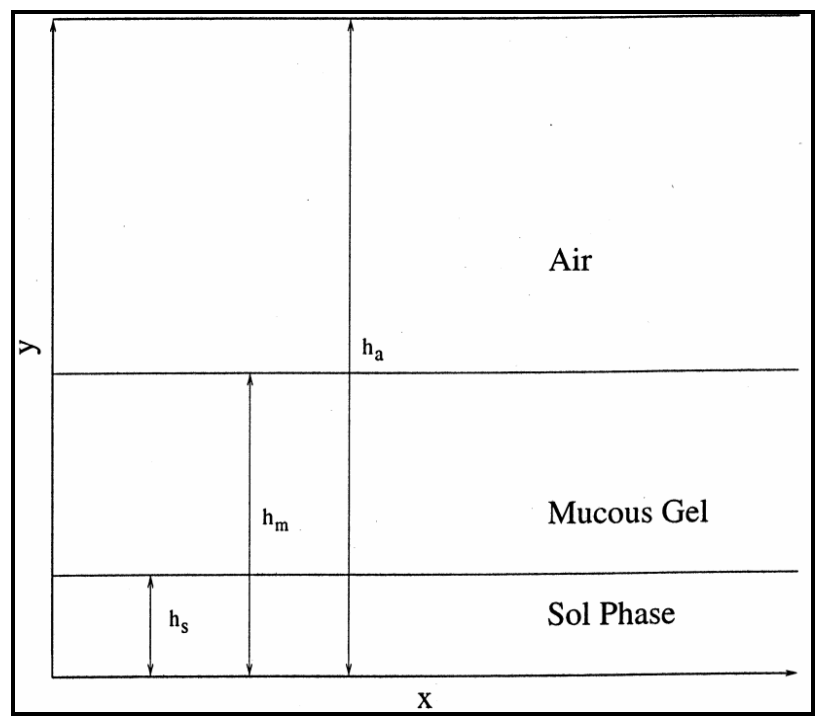

Figure 1. Flow geometry.

$$
-\frac{\partial p}{\partial x}+\frac{\partial \tau_{a}}{\partial y}=0, \tau_{a}=\mu_{a} \frac{\partial u_{a}}{\partial y}
$$

where $t$ is the time, $x$ is the coordinate in the direction of the flow, $y$ is the co-ordinate perpendicular to fluid flow, $p$ is the pressure; $u_{s}, u_{m}, u_{a}$ are the velocity components of sol phase fluid, mucous gel and air in the flow direction; $\rho_{s}, \mu_{s} ; \rho_{m}, \mu_{m}$ and $\rho_{a}, \mu_{a}$ are their respective densities and viscosities, $G$ is the elastic modulus of mucous gel and $\tau_{m}$ is the shear stress in the mucous gel layer; $\tau_{s}$ is the shear stress in the sol phase layer and $\tau_{a}$ is the shear stress in the air region. It is assumed that mucous gel behaves like a viscoelastic Voigt element whose constitutive equation is given by Equation (3) [14].

Mild forced expiration is a short time phenomena and a time dependent pressure gradient is generated in trachea. Therefore, we assume that

$$
-\frac{\partial p}{\partial x}=\phi_{p}=\phi_{0} f(t)
$$

and $f(t)$ is given by

$$
f(t)=\left\{\begin{array}{lc}
\frac{27 t}{4 T^{3}}(T-t)^{2}, & 0 \leq t \leq T \\
0, & t>T
\end{array}\right.
$$

where $t$ is the time, $T$ is the duration of mild forced expiration and $\phi_{0}$ is a constant (independent of time). The function $f(t)$ is plotted in Figure 2 for various $T$.

Since initially there is no pressure gradient, one can assume that the velocities and stresses are zero, therefore, the initial conditions are

$$
u_{s}=u_{m}=u_{a}=0, \tau_{m}=0, \frac{\partial u_{m}}{\partial y}=0 \text { at } t=0
$$

The boundary and matching conditions for the system (1) - (4) can be written as follows:

Boundary conditions:

$$
u_{s}=\beta_{s} \tau_{s} \text { at } y=0
$$

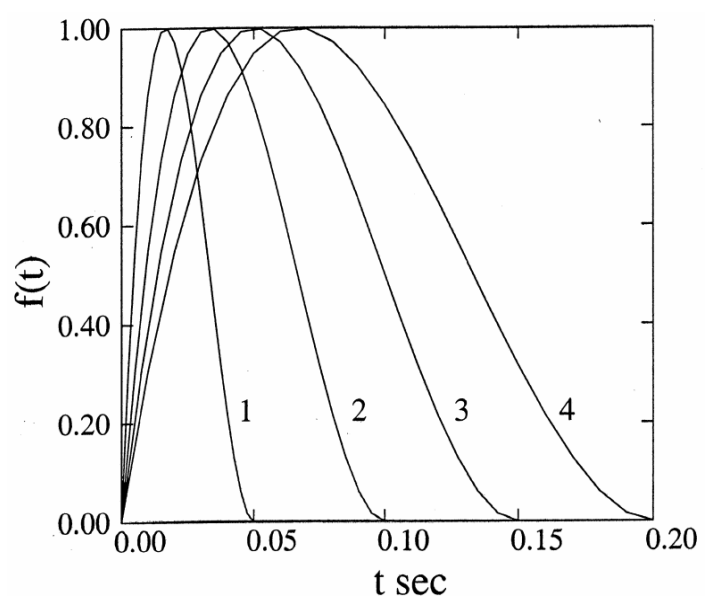

Figure 2. Graph of $f(t)$ for various $T$. 


$$
u_{a}=0 \quad \text { at } y=h_{a}
$$

Matching conditions:

$$
\begin{gathered}
u_{m}=u_{s}+\beta_{m} \tau_{s}, \tau_{m}=\tau_{s} \text { at } y=h_{s} \\
u_{m}=u_{a}, \tau_{m}=\tau_{a} \text { at } y=h_{m}
\end{gathered}
$$

In Equation (7) the right hand side represents the slip velocity at the surface $y=0$, which is caused by the slipperiness of the surfactant sol phase. Similarly in Equation (9), the second term on the right hand side represents slip velocity at the interface $y=h_{s}$ and thus the condition of the continuity of the velocities at the interface $y=h_{s}$ is still valid. $\beta_{s}$ and $\beta_{m}$ in Equations (7) and (9) are called the slip coefficients [15]. The corresponding slip velocities increase as slip coefficients increase. In a particular case, when $\beta_{s}=\beta_{m}=0$, the conditions (7) and (9) reduce to usual no-slip conditions.

\section{Calculation of Flow Rates}

Solving the Equations (1)-(4) along with the initial, boundary and matching conditions (6)-(10), the expressions for the velocity components can be found as the following.

$$
\begin{gathered}
u_{s}=U_{1} \frac{y+\mu_{s} \beta_{s}}{h_{s}+\mu_{s} \beta_{s}+\mu_{s} \beta_{m}}-\frac{\phi_{p}}{2 \mu_{s}} y^{2} \\
+\frac{\phi_{p} h_{s}}{\mu_{s}} \frac{\left(y+\mu_{s} \beta_{s}\right)\left(\frac{h_{s}}{2}+\mu_{s} \beta_{m}\right)}{h_{s}+\mu_{s} \beta_{s}+\mu_{s} \beta_{m}} \\
u_{m}=\frac{U_{1}\left(h_{m}-y\right)+U_{2}\left(y-h_{s}\right)}{h_{m}-h_{s}} \\
+\frac{\left(h_{m}-y\right)\left(y-h_{s}\right)}{2 \mu_{m}} \int_{0}^{t} \phi_{p}^{\prime}(\eta) \exp \left(-\frac{G}{\mu_{m}}(t-\eta)\right) \mathrm{d} \eta \\
u_{a}=U_{2} \frac{h_{a}-y}{h_{a}-h_{m}}+\frac{\phi_{p}}{2 \mu_{a}}\left(h_{a}-y\right)\left(y-h_{m}\right)
\end{gathered}
$$

here, $\phi_{p}^{\prime}(\eta)$ denotes the differentiation of $\phi_{p}(\eta)$ with respect to $\eta$ and the expressions for $U_{1}$ and $U_{2}$ are given by the following.

$$
\begin{aligned}
U_{1}= & \frac{a_{1}}{2} \int_{0}^{t} \phi_{p}^{\prime}(\eta) \exp \left(-a_{3}(t-\eta)\right) \mathrm{d} \eta \\
& +\frac{a_{2}}{2} \int_{0}^{t} \phi_{p}(\eta) \exp \left(-a_{3}(t-\eta)\right) \mathrm{d} \eta \\
U_{2}= & \frac{a_{4}}{2} \int_{0}^{t} \phi_{p}^{\prime}(\eta) \exp \left(-a_{3}(t-\eta)\right) \mathrm{d} \eta \\
& +\frac{a_{2}}{2} \int_{0}^{t} \phi_{p}(\eta) \exp \left(-a_{3}(t-\eta)\right) \mathrm{d} \eta
\end{aligned}
$$

where

$$
\begin{gathered}
a_{1}=\frac{\mu_{a} a_{6}+\mu_{m} a_{7}}{a_{5}}, a_{2}=\frac{G a_{7}}{a_{5}}, \\
a_{3}=\frac{G}{a_{5}}\left\{\mu_{s}\left(h_{a}-h_{m}\right)+\mu_{a}\left(h_{s}+\mu_{s} \beta_{s}+\mu_{s} \beta_{m}\right)\right\} \\
a_{4}=\frac{\mu_{s}\left(h_{a}-h_{m}\right)\left(h_{m}-h_{s}\right)\left(h_{a}-h_{s}\right)+\mu_{m} a_{7}}{a_{5}} \\
a_{5}=\mu_{a} \mu_{m}\left(h_{s}+\mu_{s} \beta_{s}+\mu_{m} \beta_{m}\right)+\mu_{m} \mu_{s}\left(h_{a}-h_{m}\right) \\
+\mu_{s} \mu_{a}\left(h_{m}-h_{s}\right) \\
a_{6}=\left(h_{m}-h_{s}\right)\left\{\left(h_{m}-h_{s}\right)\left(h_{s}+\mu_{s} \beta_{s}+\mu_{s} \beta_{m}\right)\right. \\
\left.\quad+h_{s}\left(h_{s}+2 \mu_{s} \beta_{s}\right)\right\} \\
a_{7}=\left(h_{a}-h_{m}\right)\left\{\left(h_{a}+h_{m}-2 h_{s}\right)\left(h_{s}+\mu_{s} \beta_{s}+\mu_{s} \beta_{m}\right)\right. \\
+
\end{gathered}
$$

The Volumetric flow rates per unit thickness in each of the layer are

$$
Q_{s}=\int_{0}^{h_{s}} u_{s} \mathrm{~d} y, Q_{m}=\int_{h_{s}}^{h_{m}} u_{m} \mathrm{~d} y, Q_{a}=\int_{h_{m}}^{h_{a}} u_{a} \mathrm{~d} y
$$

which after using Equations (11)-(13) can be found as

$$
\begin{gathered}
Q_{s}=\frac{h_{s}\left(\frac{h_{s}}{2}+\mu_{s} \beta_{s}\right)}{\left(h_{s}+\mu_{s} \beta_{s}+\mu_{s} \beta_{m}\right)}\left[U_{1}+\frac{\phi_{p} h_{s}}{\mu_{s}}\left(\frac{h_{s}}{2}+\mu_{s} \beta_{m}\right)\right]-\frac{\phi_{p} h_{s}^{3}}{6 \mu_{s}} \\
Q_{m}=\frac{U_{1}+U_{2}}{2}\left(h_{m}-h_{s}\right) \\
+\frac{\left(h_{m}-h_{s}\right)^{3}}{12 \mu_{m}} \int_{0}^{t} \phi_{p}^{\prime}(\eta) \exp \left(-\frac{G}{\mu_{m}}(t-\eta)\right) \mathrm{d} \eta \\
Q_{a}=\frac{U_{2}}{2}\left(h_{a}-h_{m}\right)+\frac{\phi_{p}}{12 \mu_{a}}\left(h_{a}-h_{m}\right)^{3}
\end{gathered}
$$

The average flow rates in each layer can be defined as

$$
q_{s}=\frac{1}{T} \int_{0}^{T} Q_{s} \mathrm{~d} t, \quad q_{m}=\frac{1}{T} \int_{0}^{T} Q_{m} \mathrm{~d} t, q_{a}=\frac{1}{T} \int_{0}^{T} Q_{a} \mathrm{~d} t
$$

which after using Equations (16)-(18) can be written as

$$
\begin{gathered}
q_{s}=\frac{h_{s}\left(\frac{h_{s}}{2}+\mu_{s} \beta_{s}\right)}{\left(h_{s}+\mu_{s} \beta_{s}+\mu_{s} \beta_{m}\right)}\left[U_{1}+\frac{9 \phi_{0} h_{s}}{16 \mu_{s}}\left(\frac{h_{s}}{2}+\mu_{s} \beta_{m}\right)\right]-\frac{3 \phi_{0} h_{s}^{3}}{32 \mu_{s}} \\
q_{m}=\frac{U_{1}+U_{2}}{2}\left(h_{m}-h_{s}\right)+\frac{9 \phi_{0} \mu_{m}}{16 G^{2} T^{4}}\left(h_{m}-h_{s}\right)^{3} \\
\times\left\{\frac{2 \mu_{m}}{G}\left(T-\frac{3 \mu_{m}}{G}\right)+\exp \left(-\frac{G T}{\mu_{m}}\right)\left(T^{2}+\frac{4 \mu_{m} T}{G}+\frac{6 \mu_{m}^{2}}{G^{2}}\right)\right\}
\end{gathered}
$$




$$
q_{a}=\frac{U_{2}}{2}\left(h_{a}-h_{m}\right)+\frac{3 \phi_{0}}{64 \mu_{a}}\left(h_{a}-h_{m}\right)^{3}
$$

Where,

$$
\begin{aligned}
& U_{1}=\frac{27 \phi_{0}}{8 T^{4}} \frac{a_{2}}{a_{3}}\left[\left(\frac{T^{4}}{12}-\frac{2 T}{a_{3}^{3}}+\frac{6}{a_{3}^{4}}\right)-\frac{\exp \left(-a_{3} T\right)}{a_{3}^{2}}\left(T^{2}+\frac{4 T}{a_{3}}+\frac{6}{a_{3}^{2}}\right)\right] \\
& +\frac{27 \phi_{0}}{8 T^{4}} \frac{a_{1}}{a_{3}^{2}}\left[\frac{2}{a_{3}}\left(T-\frac{3}{a_{3}}\right)+\exp \left(-a_{3} T\right)\left(T^{2}+\frac{4 T}{a_{3}}+\frac{6}{a_{3}^{2}}\right)\right] \\
& U_{2}=\frac{27 \phi_{0}}{8 T^{4}} \frac{a_{2}}{a_{3}}\left[\left(\frac{T^{4}}{12}-\frac{2 T}{a_{3}^{3}}+\frac{6}{a_{3}^{4}}\right)-\frac{\exp \left(-a_{3} T\right)}{a_{3}^{2}}\left(T^{2}+\frac{4 T}{a_{3}}+\frac{6}{a_{3}^{2}}\right)\right] \\
& +\frac{27 \phi_{0}}{8 T^{4}} \frac{a_{4}}{a_{3}^{2}}\left[\frac{2}{a_{3}}\left(T-\frac{3}{a_{3}}\right)+\exp \left(-a_{3} T\right)\left(T^{2}+\frac{4 T}{a_{3}}+\frac{6}{a_{3}^{2}}\right)\right]
\end{aligned}
$$

In a particular case, when mucus behaves as a Newtonian fluid i.e. $G \rightarrow 0$, the expressions for $q_{s}, q_{m}$ and $q_{a}$ reduce to

$$
\begin{gathered}
q_{s}=\frac{h_{s}\left(\frac{h_{s}}{2}+\mu_{s} \beta_{s}\right)}{\left(h_{s}+\mu_{s} \beta_{s}+\mu_{s} \beta_{m}\right)}\left[\frac{9 \phi_{0} a_{1}}{32}+\frac{9 \phi_{0} h_{s}}{16 \mu_{s}}\left(\frac{h_{s}}{2}+\mu_{s} \beta_{m}\right)\right] \\
-\frac{3 \phi_{0} h_{s}^{3}}{32 \mu_{s}} \\
q_{m}=\frac{3 \phi_{0}}{64}\left(h_{m}-h_{s}\right)\left[3\left(a_{1}+a_{4}\right)+\frac{\left(h_{m}-h_{s}\right)^{2}}{\mu_{m}}\right] \\
q_{a}=\frac{3 \phi_{0}}{64}\left(h_{a}-h_{m}\right)\left[3 a_{4}+\frac{\left(h_{a}-h_{m}\right)^{2}}{\mu_{a}}\right]
\end{gathered}
$$

\section{Results and Discussion}

The effects of rheological properties of mucous gel and its thickness, viscosity and thickness of sol phase fluid, slipperiness caused by surfactant sol phase and air flow rate on mucous gel flow rate are shown by plotting the expressions for $q_{m}$ given by equation. (20) in Figures 3-6( after eliminating $\phi_{0}$ with the help of equation (21)). The values of various parameters are taken as in the following [6,10,16-20].

Diameter of model trachea $\left(h_{a}\right): 1.20 \mathrm{~cm}$.

Thickness of mucous gel $\left(h_{m}-h_{s}\right): 0.05-0.20 \mathrm{~cm}$.

Thickness of sol phase $\left(h_{s}\right): 0-1.00 \times 10^{-3} \mathrm{~cm}$.

Viscosity of air $\left(\mu_{a}\right): 2.00 \times 10^{-4}$ poise .

Viscosity of mucous gel $\left(\mu_{m}\right): 1.00-10.00$ poise .

Viscosity of sol phase $\left(\mu_{s}\right): 0.01-0.10$ poise .

Elastic modulus of mucous gel $(\mathrm{G})$ :

$$
0-1.00 \times 10^{2} \text { dyne } / \mathrm{cm}^{2} \text {. }
$$

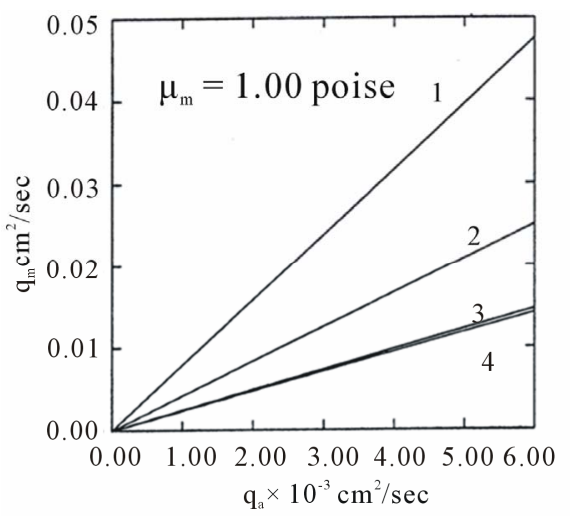

(a)

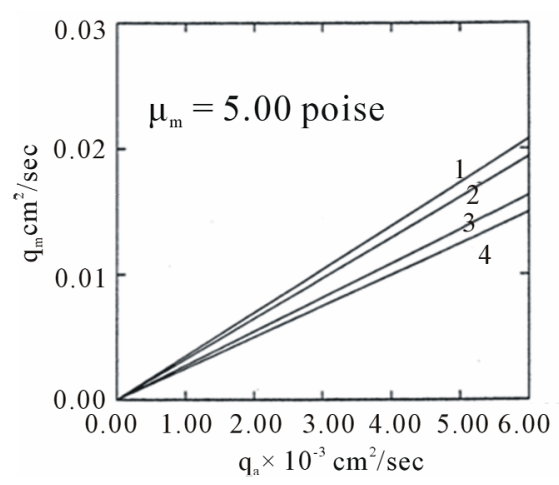

(b)

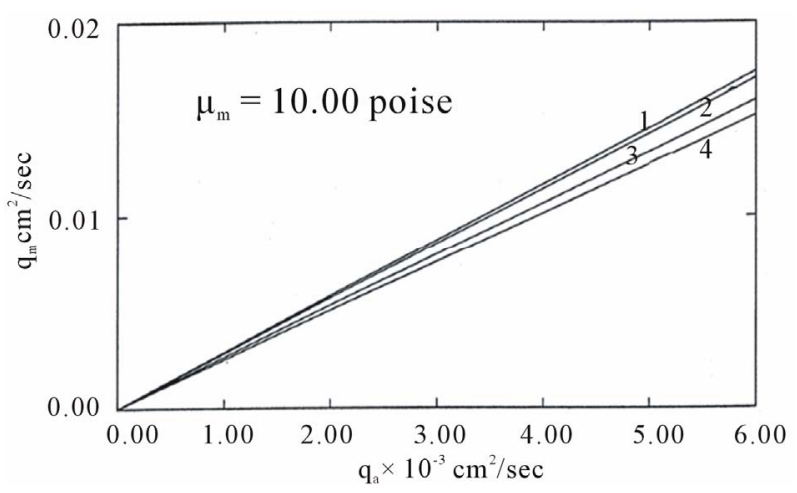

(c)

Figure 3. Variation of $q_{m}$ with $q_{a}$ for different $\mu_{m}$ and $G(1,2,3,4$ correspond to $G=0.10,50,100$ units respectively).

In our calculation, we assume

$T=0.20 \mathrm{sec}, q_{a}=0.00-6.00 \times 10^{3} \mathrm{~cm}^{2} / \mathrm{sec}$ and $\beta_{s}=\beta_{m}=0.00-0.10 \mathrm{~cm}^{2} \cdot \mathrm{sec} / \mathrm{g} \cdot \mathrm{m}$.

Figure 3 is a plot of mucous gel flow rate versus airflow rate for different $\mu_{m}$ and $G$. From this figure, it is observed that the effect of elastic modulus depends upon the magnitude of the viscosity of mucous gel. For less viscous mucous gel, Figure 3(a) shows that the gel flow rate decreases as the elastic modulus increases. This implies that the flow rate decreases when mucous gel becomes more elastic, suggesting that the efficient transfer 


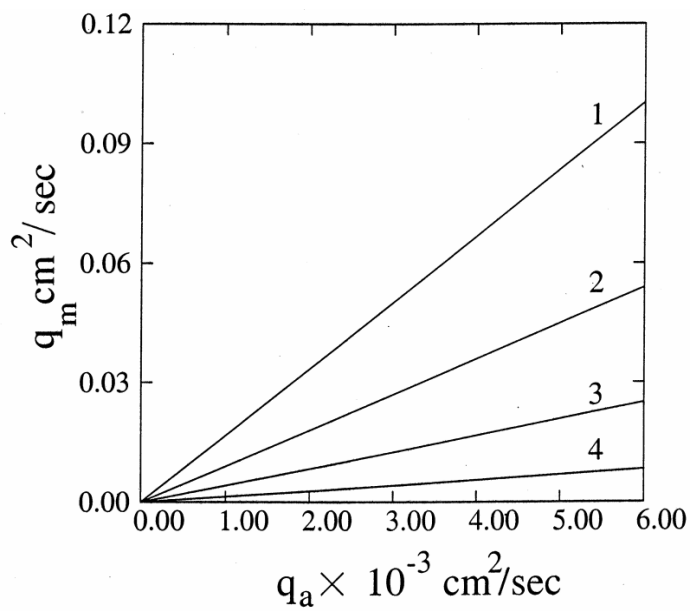

Figure 4. Variation of $q_{m}$ with $q_{a}$ for different $h_{m}-h_{s}$ (1, 2, 3, 4 correspond to $h_{m}-h_{s}=0.20,0.15,0.10,0.05$ units respectively).

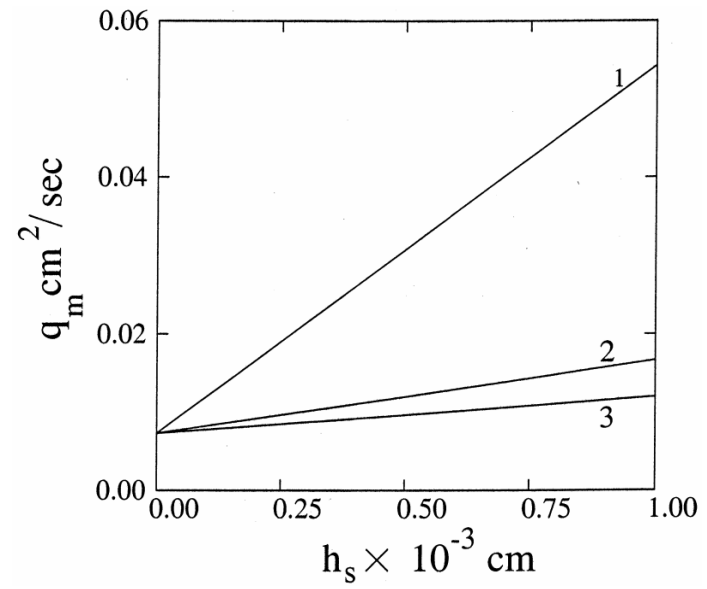

Figure 5. Variation of $q_{m}$ with $h_{s}$ for different $\mu_{s}$ (1, $2,3,4$ correspond to $\mu_{s}=0.01,0.05,0.10$ units respectively).

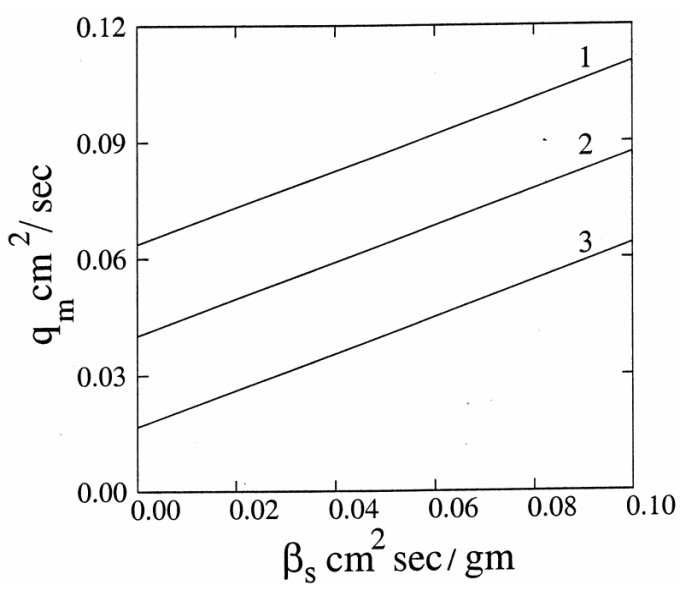

Figure 6. Variation of $q_{m}$ with $\beta_{s}$ for different $\beta_{m}(1,2$, 3 correspond to $\beta_{m}=0.50,0.10,0$ units respectively). of energy from the air flow to the mucous gel is impeded by the elastic recoil behavior of the mucous gel $[9,10]$. However for large gel viscosity, from Figure 3(c) it is observed that there is negligible effect of elastic modulus on mucous gel flow rate. Therefore, if mucous gel is represented by a Voigt element, gel transport will be more in the Newtonian case than that of viscoelastic case for less viscous mucous gel [21]. Again comparing Figures (a)-(c), it is noted that mucous gel flow rate decreases as its viscosity increases for small elastic modulus of mucous gel, but viscosity has negligible effect for large elastic modulus of mucous gel. The former result is in conformity with the experimental observations of $[7,8,11]$, who noted that gel flow rate decreases as its viscosity increases. Figure 3 also shows that mucous gel flow rate increases with the flow rate of air $[9,10]$. It may be remarked here that due to the assumption of laminar flows of air and mucous gel, only a lower limit of mucous gel flow rate is obtained by our model as in the case of simulated cough machine (where turbulent flow of air occurs).

The effects of mucous gel thickness and sol phase fluid on mucous gel flow rate are shown in Figures $\mathbf{4}$ and $\mathbf{5}$ for fixed dimensions of model trachea. From Figure $\mathbf{4}$ it is observed that mucous gel flow rate increases with its thickness. This is in conformity with the experimental observations of $[6,9,11,22]$ and analytical observations of [20,23]. Figure 5 demonstrates the role of sol phase on mucous gel flow rate. This figure shows that the gel flow rate increases as the depth of sol phase increases and this increase is further enhanced as the viscosity of sol phase decreases [6,12,13,21].

The effect of slipperiness caused by surfactant sol phase is shown in Figure 6. This figure shows that gel flow rate increases with the increase of slip coefficients. Therefore, in the presence of surfactant sol phase, the gel flow rate is more. Thus, it is reasonable to speculate that a lubricant introduced between the mucous layer and walls of the airways could increase the mucus flow rate during forced expiration. Thus, the role of surfactant is to spread and cover the entire surface of the relevant airway and thereby serve as an adhesive forming an interface or interlayer between mucus and the airway wall [24,25].

\section{Conclusion}

From the above discussion, it is concluded that the mucous gel transport increases as the slip velocity at boundary surfaces of surfactant sol phase layer increases. Therefore the role of surfactant in the mucous gel transport can be speculated as a lubricating agent acting as a serous layer and reducing the friction between the mucus layer and the surfaces of the epithelium embedded with cilia. It also observed that the gel flow rate increases with the air flow rate. For fixed airflow rate, the effect of elastic modulus on mucous gel transport depends upon the mag- 
nitude of mucous gel viscosity. For small viscosity, it increases as the elastic modulus decreases. However, elastic modulus has negligible effect on large viscosity of mucous gel. Mucous gel transport also increases with its thickness for fixed dimension of trachea and air flow rate. For fixed dimension of trachea and mucus thickness, mucous gel flow rate increases as the thickness of surfactant sol phase fluid increases for a given value of the air flow rate. This increase is further enhanced as the viscosity of sol phase fluid decreases and as the thickness of mucous gel layer increases. We hope that this study will throw some light on the role of surfactant in mucus transport in the airways due to forced expiration or cough.

\section{REFERENCES}

[1] Wanner, "Clinical Aspects of Mucociliary Transport," American Review of Respiratory Disease, Vol. 116, No. 1, 1977, pp. 73-125.

[2] L. Allegra, R. Bossi and P. Barga, "Influence of Surfactant on Mucociliary Transport," European Journal of Respiratory Diseases, Vol. 67, Suppl. 142, 1985, pp. 71-76.

[3] H. Kai, M. Saito, K. Furuswa, Y. Oda, Y. Okano, K. Takahama and T. Miyata, "Protective Effect of Surface Active Phospholipids against the Acid inducing Inhibitions of the Tracheal Mucociliary Transport," The Japanese Journal of Pharmacology, Vol. 49, No. 3, 1989, pp. 375380. doi:10.1254/jip.49.375

[4] Lachmann, "Possible Function on Bronchial Surfactant," European Journal of Respiratory Diseases, Vol. 67, No. 142, 1985, pp. 49-60.

[5] K. Rubin, O. Ramirez and M. King, "Mucus Rheology and Transport in Neonatal Respiratory Distress Syndrome and the Effect of Surfactant Therapy," Chest, Vol. 101, No. 4, 1992, pp. 1080-1085. doi:10.1378/chest.101.4.1080

[6] M. Agarwal, M. King, B. K. Rubin and J. B. Shukla, "Mucus Transport in a Miniaturized Simulated Cough Machine: Effect of Constriction and Serous Layer Simulant,” Biorheology, Vol. 26, No. 6, 1989, pp. 977-988.

[7] P. W. Scherer and L. Burtz, "Fluid Mechanical Experiments Relevant to Coughing," Journal of Biomechanics, Vol. 11, No. 4, 1978, pp. 183-187. doi:10.1016/0021-9290(78)90011-8

[8] P. W. Scherer, "Mucus Transport by Cough," Chest, Vol. 80, Suppl. 6, 1981, pp. 830-833. doi:10.1378/chest.80.6.830

[9] M. King, "The role of Mucus Viscoelasticity in Cough Clearance,” Biorheology, Vol. 24, No. 6, 1987, pp. 589597.

[10] M. King, G. Brock and C. Lundell, "Clearance of Mucus by Simulated Cough," Journal of Applied Physiology, Vol. 58, No. 6, 1985, pp. 1176-1182.

[11] M. King, J. M. Zahm, D. Pierrot, S. Vaquez-Girod and E. Puchelle, "The Role of Mucus Gel Viscosity, Spinability and Adhesive Properties in Clearance by Simulated Cough,” Biorheology, Vol. 26, No. 4, 1989, pp. 737-745.

[12] J. M. Zahm, D. Pierrot, C. Duvivier, M. King and E. Puchelle, "Influence of Airway Surface Liquid (Sol Phase) on Clearance by Cough,” Biorheology, Vol. 26, No. 4, 1989, pp. 747-752.

[13] M. Agarwal, M. King and J. B. Shukla, "Mucous Gel Transport in a Simulated Cough Machine: Effects of Longitudinal Grooves Representing Spacing between Arrays of Cilia,” Biorheology, Vol. 31, No. 1, 1994, pp. 11-19.

[14] W. L. Wilkinson, "Non-Newtonian Fluids," Pergamon Press, London, 1960.

[15] A. H. P. Skelland, "Non-Newtonian Flow and Heat Transfer,” John Wiley and Sons Inc., New York, 1967.

[16] S. S. Davis and J. E. Dippy, "The Rheological Properties of Sputum,” Biorheology, Vol. 6, No. 1, 1969, pp. 11-21.

[17] S. M. Ross and S. Corssin, "Results of an Analytical Model of Mucociliary Pumping," Journal of Applied Physiology, Vol. 37, No. 3, 1974, pp. 333-340.

[18] A. Silberberg, "Biorheological Matching: Mucociliary Interaction and Epithelial Clearance,” Biorheology, Vol. 20, No. 2, 1983, pp. 215-222.

[19] B. Yeates, “Mucus Rheology,” In: R. G. Crystal and J. B. West, Eds., The lung: Scientific Foundations, Raven, New York, 1991, pp. 197-203.

[20] D. K. Satpathi, B. V. Rathish Kumar and P. Chandra, "Unsteady Sate Laminar Flow of Viscoelastic Gel and Air in a Channel: Application to Mucus Transport in a Cough Machine Simulated Trachea," Mathematical and Computer Modeling, Vol. 38, No. 1-2, 2003, pp. 63-75. doi:10.1016/S0895-7177(03)90006-9

[21] D. K. Satpathi, "Mathematical Modeling of Mucus Transport in the Lung under Pathological and Normal Conditions," PhD thesis, Indian Institute of Technology, Kanpur, 1998.

[22] P. Camner, B. Mossberg, K. Philipson and G. Strandberg, "Elimination of Test Particles from Human Tracheobronchial Tract by Voluntary Coughing," Scandinavian Journal of Respiratory Diseases, Vol. 60, No. 2, 1979, pp. 56- 62.

[23] A. Saxena, A. P. Tyagi and R. Saxena, "Mathematical Modeling of Mucus Transport in the Lung Due to Prolonged Cough: Effect of Resistance to Flow by Serous Fluid in Cilia Bed," International Journal of Mathematical Science, Vol. 10, No. 1-2, 2011, pp. 9-20.

[24] H. Rensch and H. Von Seefeld, "Surfactant Mucus Interaction in Pulmonary Surfactant,” In: B. Robertson, L. M. G. Von Golde and J. J. Batunberg, Eds., Pulmonary Surfactant: From Molecular Biology to Clinical Practice, Elsevier Science Publishers, Amsterdam, 1984, pp. 203214.

[25] R. Banerjee and R. R. Puniyani, “Analysis of Dynamic Surface Properties of Therapeutic Surfactants and Lung Phospholipids," Journal of Biomaterials Applications, Vol. 14, No. 3, 2000, pp. 243-272. doi:10.1106/9D4B-L6PP-7CTF-BY8G 